\title{
Jonathan Dunn
}

\section{How linguistic structure influences and helps to predict metaphoric meaning}

\begin{abstract}
This paper argues that two properties of the linguistic structure of an utterance influence and partially determine whether the utterance has a metaphoric meaning that results in a stable interpretation: (i) degree of metaphoricity and (ii) degree of metaphoric saturation. A majority of metaphoric utterances in a corpus study (66\%) were unsaturated, low metaphoricity utterances that behave as expected by Max Black and the cognitive linguistics paradigm. However, a significant minority (34\%) of the metaphoric utterances were saturated or high metaphoricity utterances that behave partially as expected by Donald Davidson and others working in his tradition. This suggests that the direct and indirect interpretation views of metaphor are not incompatible but apply to different sub-groups of metaphoric utterances. The paper then constructs a model of metaphoric meaning that makes falsifiable predictions about the interpretations of metaphoric utterances in order to provide further evidence that unsaturated, low metaphoricity utterances have stable interpretations. This research provides both converging evidence for the cognitive linguistic view of metaphor and also a framework for limiting its scope to most, but not all, metaphoric utterances.
\end{abstract}

Keywords: Existence of metaphoric meaning, linguistic properties of metaphoric utterances, predicting interpretations of metaphoric utterances, behavior of lexicalized meaning during forced metaphorization

Jonathan Dunn: Purdue University, 214 Heavilon Hall, 500 Oval Drive, West Lafayette, Indiana 47904, USA. E-mail: jonathan.edwin.dunn@gmail.com

\section{The meaning of metaphors}

Do metaphors have a consistent, direct meaning; or, is there such a thing as metaphoric meaning? If metaphors do have meaning, can that meaning be modeled in such a way that predictions can be made and tested about (i) which utterances 
are metaphoric (identification) ${ }^{1}$ and (ii) what these metaphoric utterances mean (interpretation)?

There have been two sorts of answers to this question. On the one side, exemplified by Max Black, are those who say that metaphors do have a meaning, that this meaning is not simply an embellished or dressed up version of a literal paraphrase, and that users of a language follow certain rules in the creation and interpretation of novel metaphors (Black 1962, 1977). ${ }^{2}$ Instances of theories of metaphor developed using Black's archetype include Conceptual Metaphor Theory (Lakoff and Johnson 1980, 1999), which claims that metaphors have a direct meaning, that cognitive principles constrain the sorts of metaphors which are frequently used, and that the conceptual metaphors underlying metaphoric expressions can be modeled and their interpretations and inferences predicted (Narayanan 1999; Barnden 2001; Lee and Barnden 2001).

On the other side, exemplified by Donald Davidson (1978), are those who say that metaphors have a literal meaning, which is clearly absurd or false or trivial, and nothing else. Metaphor interpretation is a creative act, little bound by rules, and metaphors are subject to new interpretations with every new interpreter. What consistent meanings do exist are, perhaps, a result of inference. For example, in Martinich's (1984) approach, the final interpretation of the metaphor comes after the interpreter realizes that the literal meaning is absurd or false or trivial and, using Grice's maxims, discovers what is meant by the absurd or false or trivial statement as used in that particular situation. Metaphor cannot be modeled, in this view, because the final interpretation of the metaphor will depend entirely upon the wider context (Coulson and Matlock 2001, provide a brief juxtaposition of these two positions, together with evidence against Martinich's position).

The point of this paper is to show that both sides are partly wrong. The focus within cognitive linguistics on metaphors as cognitive mappings and blendings has disguised the amount of influence that the properties of metaphoric utterances have on whether the metaphoric expression has a consistent meaning capable of being modeled or whether it has an indirect interpretation that cannot be

1 Identification does not necessarily mean that native speakers can distinguish between metaphoric and non-metaphoric utterances. Rather, it refers to the requirement that a model of metaphor be able to identify metaphoric and non-metaphoric utterances in order to be explicit about its scope.

2 This is not the traditional narrative given within cognitive linguistics (e.g., Grady 2007) which usually starts with Lakoff \& Johnson (1980). However, it seems to me at least that Black must be credited with many of the fundamental ideas involved. Thus, I am using Black's formulation of the position. 
modeled. In other words, there is more to modeling metaphoric meaning than just cognitive mappings and blendings.

This paper has two parts. The first part, Section 2, examines Davidson's arguments against metaphoric meaning and introduces two properties of metaphoric utterances that can help us understand his arguments: the degree of metaphoricity and the degree of saturation of metaphoric utterances. These two properties influence whether an utterance can be consistently interpreted, regardless of the underlying conceptual metaphor. A corpus study shows that most metaphoric utterances (66\%) behave as we expect under the cognitive linguistics paradigm. There are, however, a surprisingly high number (34\%) of metaphoric utterances which do not necessarily behave this way. This part of the paper uncovers a number of problems with the standard approaches within cognitive linguistics that encourage the arguments that Davidson and others have made.

The second part of the paper, Section 3, builds a model and methodology for showing that, for the majority of metaphoric utterances, a prediction-making, falsifiable model of metaphoric meaning is possible. The point of this part of the paper is to provide a new source of evidence supporting the view of metaphor held by Black and the cognitive linguistics paradigm, evidence which is necessary in light of the success of arguments by Davidson for a significant number of metaphoric utterances.

This research has two significances for metaphor research within cognitive linguistics. First, it shows the importance of attention to linguistic form in addition to cognitive mappings and blendings. In other words, the structure of the utterance makes a difference. For example, Falck and Gibbs (2012: 258) point out that:

Cognitive linguistic research on metaphor has not generally focused sufficiently on the role of linguistic meaning in the creation and continued use of metaphorical concepts, because of its strong emphasis on the experiential grounding of linguistic structure and behavior.

The linguistic structure of metaphoric utterances discussed here is one source of the linguistic meaning that can eventually influence metaphoric concepts, suggesting a mutual dependence. Second, this research protects work on metaphor within cognitive linguistics from the sorts of arguments used by proponents of philosophical and formal semantics to dismiss linguistic research on metaphor. This is important because while Kértesz and Rákosi (2009) and Ruiz de Mendoza and Perez Hernandez (2011) defend approaches to metaphor within cognitive linguistics against criticisms of their internal theoretical constructs, they do not 
consider criticisms of their premises (that metaphoric utterances have a stable meaning) ${ }^{3}$ or their scope (that all metaphoric utterances fall under the theory's description).

\section{The influence of linguistic structure on metaphoric meaning}

\subsection{Arguments against metaphoric meaning}

Davidson (1978: 31) begins by saying that:

Metaphor is the dreamwork of language and ... its interpretation reflects as much on the interpreter as on the originator.... So too understanding a metaphor is as much a creative endeavor as making a metaphor, and as little guided by rules. ${ }^{4}$

Another way of putting this is that users of a language lack a competence for consistently interpreting metaphoric utterances (whether that competence is linguistic or otherwise) because there is no such thing as metaphoric meaning to be interpreted. Metaphors have only an absurd or false literal meaning which requires that each act of interpretation be independent and unique, a creative act of making meaning where no meaning existed before. ${ }^{5}$ Interpretation is a sort of production.

In the following paragraph he adds, "there is no manual for determining what a metaphor 'means' or 'says.' ”' (Davidson 1978: 31). He goes even further in the first footnote by disagreeing with Black's (1962: 29) statement that the rules of

3 This is a premise of CMT in the sense that conceptual metaphors and domain mappings and embodied experience are used to explain how metaphors have this stable meaning.

4 This sort of interpretive-creativity is very different from the productive-creativity in Kövecses (2010). In fact, the two are inversely related in the sense that if Davidson is right about the proliferation of interpretive-creativity then productive-creativity is not particularly useful: no one will be capable of understanding the results of that productive-creativity.

5 Grady (2007: 196) argues briefly that Davidson sees possible metaphoric connections as unconstrained, with no limits on what conceptual metaphors are possible, and that he "goes so far as to claim that metaphors have no meaning." Grady has the order of these positions backward: Davidson sees metaphors as utterances without a direct (non-inferential) meaning, and from this it follows that certain metaphoric meanings cannot be preferred over others. Grady also overlooks the fact that Davidson is right about many metaphors, which do not have a stable interpretation and in this way seem unconstrained. 
language determine what must count as a metaphoric utterance. ${ }^{6}$ For Davidson, there is no consistent way to identify metaphoric utterances because there are no properties that separate them from other false or absurd utterances. This represents the skeptical position about metaphors, and figurative language more broadly, that they have neither a systematic meaning nor a systematic character of any kind. He argues, rather, that metaphoric utterances do not exist as such.

Davidson's main thesis is that metaphoric utterances do not have some special metaphoric meaning but only their literal meaning. His argument is that although the interpreters of the metaphoric utterance do receive some sort of "meaning" from the utterance containing the metaphor, this meaning is not present in the utterance itself (somehow encoded or secreted inside it) but rather is present only in the interpreter. Thus, with each situation and each creative interpreter, the metaphor can have a different "meaning." Metaphor is entirely in the eye of the beholder. The reason it is so difficult to interpret even the simplest metaphors is that metaphors do not have a meaning to be interpreted. The evidence that metaphors do not have a stable meaning, then, is that we know no easy or straightforward way to get at that meaning. If it existed, we would have found it (for a practical counter-argument, see Section 3).

Davidson is partly right and partly wrong. Davidson's argument goes wrong because it turns out that metaphoric utterances come in many different shapes and sizes. While I see merit in Black's original reply (1979) to Davidson, one of its weaknesses is that he does not admit that Davidson's argument does, in fact, work for some cases: metaphors which lack a direct meaning (what Davidson calls a literal meaning) and, as a result, trigger a series of inferences no different from those present in non-metaphoric utterances which fail to meet the conversational maxims. However, metaphors like this are a minority of metaphoric utterances. They are not representative of the sorts of metaphors actually used in texts and they are by no means numerically the most frequent (see corpus study below). In other words, Davidson incorrectly generalizes a valid argument about a small sub-set of metaphors to all metaphors. Most metaphors have a stable interpretation derived from a direct meaning or, as Black says, "to be able to produce and understand metaphorical statements is nothing much to boast about" (Black 1979: 131).

In making this argument, I am not engaging directly with the debate about whether metaphor has meaning as something which is said directly and "directly

6 Black's position is more nuanced than Davidson's representation of it, however. Later in the same paragraph which is quoted by Davidson, Black writes, "This is an example ... of how recognition and interpretation of a metaphor may require attention to the particular circumstances of its utterance." (Black 1962: 29). 
expresses a proposition, which can potentially be evaluated as either true or false" (Bezuidenhout 2001: 156; also, Wearing 2006), or whether metaphor has meaning as constructed by the hearer based on inferences or pragmatic principles and "not expressed by the words themselves" (Reimer 2001: 152; also, Martinich 1984; Black 1979: 138 puts Davidson in this category). This debate is somewhat simplistic because metaphoric utterances are not all one or the other: metaphoric utterances can have either direct meanings or indirect interpretations. Metaphors are no different in this respect than non-metaphors. The debate between direct and indirect metaphoric meaning is misplaced because this is not an issue solely for metaphors but for all utterances. In short, the fact that an utterance is metaphoric has no bearing on whether its meaning is directly or indirectly interpreted, so that the two positions are not necessarily opposed (this differs from Coulson and Matlock 2001, who see the two positions as always in contrast).

\subsection{Variations in metaphoricity}

Linguistic properties cause some utterances to be more metaphoric than others (Dunn 2011; Coulson and Matlock 2001, among others, focus on non-linguistic causes of the same property). There are theoretical accounts elsewhere of why this is the case; for my purposes here I simply want to show that it is the case. Let's look at some examples. In (1a), we see an entirely non-metaphoric utterance. In (1b), the verb is changed to demolished, making the utterance mildly metaphoric. In (1c), the patient case role is also changed to John's stronghold, making the utterance more metaphoric still. In (1d), the instrument case role is also changed to new found weapon; this utterance is more metaphoric than the others (it is also a saturated metaphoric utterance, as we will see shortly). My point here is that metaphoricity varies continuously, with some utterances being more and others less metaphoric (see also Evans 2010).

(1a) Mary disproved John's argument with her newly found evidence.

(1b) Mary demolished John's argument with her newly found evidence.

(1c) Mary demolished John's stronghold with her newly found evidence.

(1d) Mary demolished John's stronghold with her newly found weapon.

I would also like to observe that there is no clear dividing line between non-metaphoric and metaphoric. Thus, while some utterances are clearly nonmetaphoric and others are clearly metaphoric, there is also a large third category which is ambiguous between the two (in other words, it is hard to tell if such ut- 
terances are metaphoric or not). Thus, in (2a) this description of a company's profit report is clearly non-metaphoric. And, in (2b), this description of the same report is clearly a metaphoric utterance. However, the description in (2c) is not so clearly one or the other; rather, it seems to straddle the boundary between nonmetaphoric and metaphoric. This is an important point because to say that metaphoric utterances have only a literal meaning (a premise essential for Davidson's argument) assumes that such a literal, non-metaphoric meaning is clear-cut and not problematic.

(2a) Company profits continued to increase during the third quarter.

(2b) Company profits soared to new heights during the third quarter.

(2c) Company profits rose steadily during the third quarter.

\subsection{Saturated and unsaturated metaphoric utterances}

Metaphoric utterances can be divided into two categories, saturated and unsaturated, based on their utterance-internal relations (Dunn 2011). The degree of saturation depends upon how much of the utterance is filled with metaphoric material. Unsaturated utterances contain elements from both the source and the target domains. Saturated utterances, on the other hand, contain elements from only the target domain. This means that they could be either entirely non-metaphoric or entirely metaphoric depending only on the wider context. Let's consider the utterance in (3a), repeated from (1d) above. This sentence is saturated and cannot be ambiguous between metaphoric and nonmetaphoric, but rather can only be entirely metaphoric (when followed, for example, by [3b]) or entirely non-metaphoric (when followed, for example, by $[3 c])$.

(3a) Mary demolished John's stronghold with her new found weapon.

(3b) He had to agree that her theory was better formed.

(3c) He had to flee and leave his material possessions behind.

Now let's look at the low metaphoricity utterances from (1) above, both of which are unsaturated. These contain elements from both the source and target domains. This means that the utterance is connected to both primary and secondary cognitive models (Evans 2010). Metaphorically speaking, there is a clash between these different models. To use a different metaphor, if we assume that natural language utterances have a semantic structure that consists, in part, of case role organization connecting an event with its arguments (Fillmore 1967), 
and if we assume that the arguments of an event must meet certain selectional restrictions (Katz and Fodor 1963), then the utterances in (4a) and (4b) contain mismatched arguments. ${ }^{7}$ In other words, some of these case role fillers would not meet the selectional restrictions, because in non-metaphoric language ARGUMENTS are not physical objects capable of being demolished, and EVIDENCE is not a physical instrument capable of demolishing strongholds. Unsaturated utterances like this can be identified as metaphoric in any context with reference only to their structure. This is because there is a mismatch or divergence between the elements of the utterance, which come from both source and target domains.

(4a) Mary demolished John's argument with her newly found evidence.

(4b) Mary demolished John's stronghold with her newly found evidence.

We can see, then, that only saturated metaphoric utterances have the possibility of having both a non-metaphoric and metaphoric meaning. To be clear, I am not arguing that saturated utterances are ambiguous between non-metaphoric and metaphoric because the lexical items involved are ambiguous between a non-metaphoric and a metaphoric meaning. Rather, I am saying that saturated utterances (and only saturated utterances) can be either non-metaphoric or metaphoric as a whole based on their use in a particular context. This is important because Davidson's argument depends on the idea that metaphoric utterances have a false or absurd literal or non-metaphoric meaning. Only saturated metaphoric utterances, however, have something that could be called a literal meaning.

\subsection{Metaphors with no stable interpretation}

The following examples come from the Corpus of Contemporary American English (Davies 2008; henceforth COCA). The utterance in (5) has high metaphoricity (in this case because of a highly metaphoric cross-domain mapping). The utterance does not have a stable interpretation. The same is true for the utterance in (6). What does it mean to "invest brainpower in looks"? The interpretation requires the creativity of each interpreter, as Davidson argues, and a range of interpretations is possible.

7 These assumptions are not necessary for the empirical point being made; I am using this particular model here only because it clarifies the point in question. 
(5) But as I have long maintained, poetry is something encoded on one's soul, and it is a lucky few who are able to decipher it during their lifetime.

(6) A lady on high heels clacked along, the type my mother says invests all of her brainpower in her looks.

In (7) the utterance again has high metaphoricity and lacks a single interpretation. Does Lorrain herself use different disguises or does she read through different disguises? Does she often return to old disguises? The interpretation is not fixed. Likewise, in (8) the interpretation is not clear and the interpreter must use something like Martinich's (1984) approach to make sense of the utterance.

(7) The proliferating disguises and pen names through which Lorrain would constantly migrate attest to a need for self-fictionalization experienced as travel and change.

(8) The seasons have married into this gumbo culture that's something that people love to come visit.

Similarly, the metaphoric utterances in (9), (10), and (11) have high metaphoricity. The utterances lack a consistent or stable interpretation and in this way show evidence that metaphor is sometimes the "dreamwork of language." How does the Bible create a world by imagining it? What does that mean? In what way does the writer offend beauty by writing about it? Does offending the hand involve injuring the hand? Or simply being inconvenient (this is also metonymy)?

(9) I don't think the Bible describes our world; the Bible imagines it, and by imagining it creates a world drenched with grace from God.

(10) Beauty, by its nature, is agreement. I want to offend beauty by writing about it.

(11) Their hatchback lets you easily load. Their interiors fit together well and offend neither eye nor hand.

I will turn now to saturated utterances. The utterance in (12) below is saturated in the sense that all of its structure is filled with material compatible with the metaphor: we cannot tell if Jack Kemp is being removed from a list or, perhaps, killed. The first could be non-metaphoric, but the second would be metaphoric. Thus, this could be a non-metaphoric sentence or, in the context of (12a), could be used to refer to murder and have high metaphoricity. Similarly, the utterance in (13) could be either a saturated and high metaphoricity 
utterance discussing someone's performance in school (it is), or it could be a nonmetaphoric discussion of someone's performance during a race. Thus, there is again no stable interpretation that does not depend upon the larger discourse context.

(12) Well, this week we subtracted Jack Kemp, which is really kind of unfortunate.

(12a) We didn't want to kill Jack.

(13) At a meeting in early March, Crewe told them "the high bar we want you to jump now is the one you should have been jumping all along. The problem is that you've been trained to jump too low."

Again, in (14) we do not know if this is a metaphoric utterance, for example if it means that he can make otherwise sophisticated people leave an investment in a hurry and thus lose out on upcoming gains, or if it is a non-metaphoric utterance about a practical joker. In (15) we do not know whether this is a metaphoric utterance in which people are emotionally invested or have invested time in losing weight (and thus, why make changes when they will soon lose weight?) or whether it is a non-metaphoric utterance talking about financial profits (will making things easier for the overweight hurt gym memberships?).

(14) He can make fools of his well-educated employers and even his billionaire father-in-law: he can cause these otherwise sophisticated people to jump, and then laugh at them behind their backs.

(15) Oakland-based Lyons consults with doctors and club owners who want to create more welcoming environments for large women. But she admits it's hard to change preconceptions, especially when so much is invested in weight loss.

This is evidence that both high metaphoricity and saturated metaphoric utterances do not carry a stable or consistent interpretation. High metaphoricity utterances (like most metaphors) have only a metaphoric meaning; but that metaphoric meaning has many possible interpretations. Saturated utterances (and only saturated utterances) have the possibility of both a non-metaphoric and metaphoric meaning that Davidson takes for granted. As a result, the interpreter must decide whether to take the saturated utterance as metaphoric or nonmetaphoric, a process that may or may not take place in the way Martinich describes.

Even though these two kinds of metaphoric utterances lack a stable interpretation as Davidson argues, they behave differently (something not described by Davidson's approach). Saturated utterances lack a consistent interpretation only 
when viewed in a limited context, but high metaphoricity utterances lack a consistent interpretation in all contexts.

\subsubsection{Counter-examples}

Does this mean that all high metaphoricity and saturated utterances lack a stable interpretation? Interestingly, no. The counter-example in (16a) below shows an utterance with many metaphoric constituents (and so high metaphoricity) which is still interpretable. If we remove in their relationship, as shown in (16b), the result is an unsaturated utterance. In this case, the utterance now allows both a metaphoric and a non-metaphoric interpretation. One possible answer is that the version in (16a) uses conventionalized material, with the result that it does not actually have high metaphoricity. What happens if we exchange this material with less conventional manifestations of the same conceptual metaphor? The version in (16c), based on the same cross-domain mappings but differing in the linguistic realization of those mappings, does not share the same stable interpretation as (16a). This suggests, then, that linguistic structure, in addition to crossdomain mappings, influences the interpretation of the metaphoric utterance.

(16a) They were at a cross-roads in their relationship, so they decided to turn back on their tracks and find a better way to get where they wanted. ${ }^{8}$

(16b) They were at a cross-roads, so they decided to turn back on their tracks and find a better way to get where they wanted.

(16c) They were at a big intersection in their relationship, so they decided to do a U-turn and find a way to get where they wanted that had less speed bumps.

\subsection{Metaphors with a stable interpretation}

Metaphors contained in unsaturated or low metaphoricity utterances have a direct meaning with a stable interpretation. By direct meaning, I intend to say that there is no false literal meaning because the only meaning is the metaphoric meaning. For example, the utterance in (17a) does not depend upon conversational implicatures nor upon the creative interpretation of the hearer for its meaning. The hearer will not first construct a non-metaphoric meaning, reject that

8 Thanks to an anonymous reviewer for this example. 
meaning, and use Grice's maxims to construct a situational interpretation. This is because the utterance, although a metaphor, possesses what I am calling a direct meaning. The same is true for (17b) and for (17c) and for (17d). We could go on giving examples, but the point is that these and most metaphoric utterances, for whatever reason and from whatever source, do carry a stable interpretation for speakers of English.

(17a) Mary demolished John's argument with her newly found evidence.

(17b) Company profits soared to new heights during the third quarter.

(17c) Mary's theory had a firm foundation.

(17d) Her marriage was running out of fuel.

The situation, then, is that the properties of metaphoricity and saturation, together with properties of the underlying cross-domain mappings, determine whether a metaphoric utterance will have a direct meaning allowing a stable interpretation (a direct meaning because there is no absurd or false literal meaning which must first be discarded) and be described by Black's work and work within cognitive linguistics, or whether it will have only an indirect interpretation (indirect because there is the possibility for a non-metaphoric meaning and so the interpretation must be worked out by inference or because there are multiple metaphoric interpretations possible) and be described by Davidson's work.

\subsection{Corpus evidence: Frequency of saturation and metaphoricity}

Which type of metaphor is more common? A corpus study was conducted to determine the frequency of saturated and unsaturated metaphoric expressions and of low metaphoricity and high metaphoricity expressions in American English using COCA.

\subsubsection{Methodology}

The study first selected 20 verbs, 5 each from the general domains PHYSICAL, MENTAL, SOCIAL, and ABSTRACT. Although some of the verbs could not be discretely categorized into only one domain, this approach was used to ensure a wide representation of metaphors. Some of the domains, such as MENTAL, yielded many fewer metaphoric expressions than others, such as PHYSICAL. The domains were equalized by choosing 25 tokens of each verb for a total of 125 tokens 
of each domain and a total of 500 metaphoric expressions. A random sample of each word was drawn from COCA and metaphoric expressions were included in the study as they were encountered. The intuitions of the author were used to identify metaphoric vs. non-metaphoric expressions. The complete verb list can be found in Appendix 1.

The 500 metaphors in the study were randomly sorted and then annotated for two features: saturation and metaphoricity. Each feature was given two values. For saturation, 0 was used for unsaturated and 1 for saturated expressions. For metaphoricity, 0 was used for low metaphoricity and 1 was used for high metaphoricity. A precise procedure for determining high metaphoricity does not currently exist (although see Dunn 2011 for work in that direction). The annotations erred on the side of inclusion in questionable cases.

\subsubsection{Results}

As shown in Table 1, only $13 \%$ of the metaphors were saturated. Only $21 \%$ were high metaphoricity. This means that, overall, $66 \%$ of the metaphors were neither saturated nor high metaphoricity and thus not subject to Davidson's arguments (however, see Section 2.7 below for a discussion of methodological decisions that made the other 34\% perhaps higher than it should be). On the one hand, this means that Davidson is mostly wrong. On the other hand, this means that Davidson is right about quite a few metaphors. The problem is that most theories of metaphor do not take these distinctions based on the linguistic structure of the metaphoric utterance into account, even though the two types of metaphors behave very differently.

There is something else to notice in these results: the prevalence of saturated utterances differs between domains. The difference between domains in saturation was significant (at the $\mathrm{p}=.11$ level); the difference between domains in metaphoricity was not significant (each using a chi-square test of frequencies). There was also a significant difference between the domains in terms of whether the metaphors have a consistent interpretation (e.g., between unsaturated, low

\begin{tabular}{lccccr} 
& Physical & Mental & Social & Abstract & \multicolumn{1}{c}{ Total } \\
\hline Saturated & $34[27 \%]$ & $01[00 \%]$ & $09[01 \%]$ & $23[18 \%]$ & $67[13 \%]$ \\
High metaphoricity & $23[18 \%]$ & $17[14 \%]$ & $33[26 \%]$ & $33[26 \%]$ & $106[21 \%]$ \\
Both & $00[00 \%]$ & $00[00 \%]$ & $00[00 \%]$ & $02[00 \%]$ & $02[00 \%]$ \\
Neither & $68[54 \%]$ & $107[86 \%]$ & $83[66 \%]$ & $71[57 \%]$ & $330[66 \%]$ \\
\hline
\end{tabular}

Table 1: Frequency of Metaphoricity and Saturation 
metaphoricity utterances and all others; at the $\mathrm{p}=.01$ level). I did not expect these significant differences to occur. Certainly, there were many more metaphors in the PHYSICAL domain than in the MENTAL domain, but in the results above there was a fixed number of tokens from each domain in order to overcome that disparity. I will present no explanation for this here, except that it suggests that not only are the frequencies of metaphors constrained by cognitive mappings, but that those same mappings influence the presence of these linguistic properties of metaphoric utterances. This implies a mutual dependence.

\subsection{Causes of saturation}

There are a few properties of a metaphoric utterance that can cause it to be saturated (I am using 'cause' in a loose sense because I mean that saturated utterances have some or all of these other properties). First, referential ambiguity in one of the arguments can leave the verb open between non-metaphoric and metaphoric interpretations, as in (18a) below (this and the following examples are taken from COCA). This causes saturation in a corpus study that restricts its attention to smaller units of language, but in discourse these saturated metaphors will disappear (because the reference will not be ambiguous). The same is largely true of saturated metaphors caused by lexical ambiguity, as in (19a) below. Again, in a larger context (although not in the corpus study above) these ambiguities will likely be resolved and the saturation will disappear.

(18) Referential ambiguity

(18a) I didn't know what it was. It would not compute. It had a kind of clarity about it.

(19) Lexical ambiguity

(19a) So - and they can't throw the case out.

Unspecified arguments are a more persistent cause of saturation. In (20a) we can assume that what is jumping is the number of chapters and conferences. But it isn't specified. The same is true in (20b), where the missing argument will determine whether or not the utterance is metaphoric.

(20) Unspecified arguments

(20a) India, the number of members increased 18\% and chapters and conferences also saw significant jumps.

(20b) Mom butchered ours. There's only two pictures left of Dad in all twelve albums. 
Background knowledge or world knowledge can also cause saturation because the utterance itself is unspecified to some degree. For example, in (21a) the town is not legally owned. But, without background knowledge filling in this information, a non-metaphoric interpretation is possible.

(21) Background Knowledge

(21a) It's about a town bought and paid for and subverted by a gangster element.

\subsection{Causes of high metaphoricity}

Utterances that are high metaphoricity tend to have some or all of the following properties. The first is that these utterances have multiple metaphoric constituents (this also means that they lean toward saturation). For example, in (22a) there are several metaphoric constituents in the utterance (from different metaphors), which makes the utterance as a whole more metaphoric. While saturation tends to occur when an utterance is filled completely with a single metaphor, high metaphoricity results also when there are multiple metaphors in the utterance.

(22) Many metaphoric constituents

(22a) Veterans of many an evening at the routine pursuit of duty in the field, their emotional sacs barnacled with cynicism and their minds programmed for sardonic wit, sprang to their feet.

The underlying metaphor can also give an utterance high metaphoricity (something frequently researched, for example with Black's resonant metaphors, and in Coulson and Matlock's 2001 study that involved a continuum between non-metaphoric and metaphoric, and in Evans' LCCM Theory 2010). In (23a) it is, in part, the properties of the underlying metaphor which makes the utterance high metaphoricity. In (23b), similarly, the reverse of the more common conduit metaphor results in a high metaphoricity utterance. This is not so much a linguistic property as a property of metaphor-in-thought.

(23) High metaphoricity connection

(23a) I don't marry myself to one belief system.

(23b) Poetry is something encoded on one's soul, and it is a lucky few who are able to decipher it during their lifetime.

The presence of grammatical metaphors can also result in high metaphoricity. For example, in (24a) the utterance has higher metaphoricity than the 
constructed counterpart in (24b), although the only difference between them is the linguistic structure. Further, both are equally saturated. For this reason, it seems that unusual constructions (or argument structure) can increase metaphoricity. This fits with Lakoff's view of grammar (1987) in which some constructions are metaphorical in themselves. In other words, high metaphoricity utterances can result when a metaphor is expressed using a metaphorical construction, compounding the presence of metaphor in the utterance.

(24) Altered or unexpected argument structure

(24a) My normal sensations flooded back, although I had to migrate my eyes to get them pointed forward.

(24b) My normal sensations flooded back, as my eyes migrated forward again.

\subsection{Applications to metonymy}

Metonymic utterances can also be saturated, meaning that in isolation the utterance is ambiguous between a metonymic and non-metonymic interpretation. For example, the utterance in (25a) could have either the interpretation in (25b) or that in (25c).

(25a) The ham sandwich is getting cold.

(25b) The man who ordered the ham sandwich asked to have the heat turned up.

(25c) The ham sandwich that was prepared is getting cold and needs to be brought out.

Similarly, metonymic utterances vary in how strikingly metonymic they are. For example, some cases of metonymy have stronger metonymic links than others. The examples in (25) are different metonymic sources to the same target. The example in (25a) points out the customer's order, (25b) points out the customer's location, and (25c) points out a physical attribute of the customer. I will not speculate on the factors that influence the strength of these links here (but see Panther and Thornburg 1998).

(25a) The ham sandwich wants his coffee now.

(25b) Table 6 wants his coffee now.

(25c) The red shirt wants his coffee now.

The argument that metaphors have only a literal meaning that is either false or absurd and thus incapable of being false reaches further problematic 
ground with metonymy: do these utterances have a literal meaning in addition to a metonymic meaning? In most cases, they do not. The issue here is more difficult than with metaphor because of the conceptual contiguity of metonymy (Dirven 1993) which causes metonymy to be defeasible (and thus more likely to be saturated). Whether these utterances have a direct meaning with a stable interpretation or an indirect interpretation resulting from a rejected literal meaning will also vary, at least in part, according to the linguistic properties of the utterance.

\subsection{Conclusions}

The arguments in this part of the paper problematize current approaches to metaphor by showing that the linguistic structure of metaphoric utterances has a large influence on whether the metaphor has a direct and consistent meaning or an indirect interpretation dependent on the situation of its use. The first category of metaphors can be modeled, as in CMT, but the second category cannot (or at least, not in the same way). While the first category is the largest, it is not so large as I, at least, had expected. This leads to an unexpected critique of approaches to metaphor within cognitive linguistics, that they attempt to model what cannot be modeled because it does not properly exist in all situations (e.g., metaphoric meaning). In order to prevent this critique from being taken too far, I will now present a new source of evidence that some metaphors have the sorts of meanings that are described by work in cognitive linguistics.

Why is this necessary when there is already a great deal of processing evidence against the reinterpretation views of Martinich (1984) and Searle (1979), for example Coulson and Matlock (2001) and the survey in Evans (2010)? First, Davidson's arguments against the existence of metaphoric meaning do not necessarily require the reinterpretation view. In other words, that view arose in part because the position that metaphors are meaningless goes against the common use and interpretation of metaphoric expressions, so that some explanation of how meaningless utterances come to have meaning was necessary. Second, neither of these two positions make any testable predictions about processing, in their view (unlike the view within cognitive linguistics) a separate issue. Third and most importantly, processing is in many ways tangential to the issue, which is whether metaphors have a direct meaning with a stable interpretation. Processing does not tell us what the interpretation is. We need a way to show that interpretations are stable; the surest way to do this is to make and test predictions about metaphoric meaning. 


\section{Falsifiable predictions of the meaning of metaphors}

\subsection{Using forced metaphorization to predict changes in utterance meaning}

In Popper's (1959[1934]) view of scientific theories, a true theory must be falsifiable. In other words, the constructs required for a scientific theory cannot themselves be individually observed and tested, so the theory as a whole is tested by determining what predictions it makes, what its expectations are, and then testing those expectations. This part of the paper presents a methodology for making testable predictions about the interpretation of metaphoric utterances. If we can make valid predictions about the interpretation of metaphoric utterances then it means that those interpretations are relatively stable. ${ }^{9}$

My argument hinges upon the claim that we can predict what meaning change will occur when we force metaphorization upon a non-metaphoric utterance. In other words, we can take a non-metaphoric utterance and substitute into it a single lexical item that forces the utterance to become metaphoric. This creates an unsaturated metaphoric utterance. For each such forced metaphorization we are left with a created metaphoric utterance. If we substitute multiple lexical items into the original non-metaphoric utterance, then we can compare the meanings of each of the resulting metaphoric utterances. My argument is that we can predict whether two substituted lexical items will cause the same or different changes in meaning using only their lexicalized meanings (in particular, whether they point to the same or different concepts). I am using forced metaphorization because it allows us to make testable predictions about what the interpretation of the resulting metaphoric utterance will be.

In order for this to work we need to know two things: (i) the lexicalized meaning of each of the substituted lexical items and (ii) the meanings of the created metaphoric utterances. We can use (i) the lexicalized meaning of the lexical items to predict (ii) whether they will cause the same or different meaning changes in the metaphorized utterance. We can make these predictions because

9 To reformulate this in the terms used by Kértesz and Rákosi (2009), the failure to falsify these predictions will lead to a plausible inference that these metaphoric utterances have a stable, direct meaning. At the same time, negative falsification evidence is stronger than positive plausible inferences, although it does require its own plausible inferences involving (i) what the predictions of the model actually are and (ii) what sources of evidence and what cases count as falsifying. 
these sorts of metaphors (low metaphoricity and unsaturated) have a stable interpretation.

There are two matters that we must consider before we can make such predictions about the interpretation of metaphoric utterances: (1) different ways that meaning is lexicalized (so that we know when lexical items point to the same or different concepts); and (2) ways to determine what changes in meaning result from forced metaphorization.

\subsubsection{Semantics and pragmatics, propositional and non-propositional meaning}

The constructs of propositional and non-propositional meaning are problematic because their definitions often rest on the non-existing distinction between semantic and pragmatic meaning. There are many different approaches to meaning, but through them all there remains the observation that some sorts of meaning are more important, more essential, more stable than other sorts of meaning. The term "propositional" refers directly to the logical formalism used for representing the sense or intension of an utterance in formal semantics. The narrow use of the term includes only declarative statements that have a truth-value. The broader use of the term, however, includes all stable or essential or nondefeasible linguistic sources of meaning. For those who adhere to Davidson's position on metaphor, metaphoric meaning is non-propositional (in the narrow sense) because metaphors are literally false or absurd (and thus incapable of being false). I have argued above that this view of metaphoric meaning is incorrect for most metaphoric utterances. Thus, although I think that the term "propositional meaning” in the broad sense provides a useful distinction, I will choose a different set of terms to make the distinction between non-defeasible, essential, direct meaning and defeasible, descriptive, secondary meaning. (I am excluding connotations or heteroglossia effects (Bakhtin 1994 [1930s]) from this discussion entirely.) In what follows, I will refer these two different sorts of meaning using the terms "essential meaning" and "non-essential meaning." This distinction does not depend on the difference between semantic or pragmatic meaning. While it remains problematic, no unproblematic alternative is available.

\subsection{Lexicalized meaning}

Research on lexicalization has shown that any semantic property of an utterance (i.e., any part of the utterance meaning) can be expressed in the explicit linguistic structure or can be expressed internally within a single lexical item. In other words, there is a continuum between grammar and the lexicon (with no discrete 
boundaries between them) and both points of this continuum can be used to express meaning. Lexicalization in this context refers to the inclusion of meaning in a lexical item. For this study I draw from the tradition of ontological semantics (Nirenburg and Raskin 2004; henceforth OnSe), which represents lexical meaning by separating concepts and the lexical items which (a) point to concepts and (b) lexicalize additional properties while pointing to concepts. ${ }^{10}$ Thus, many lexical items point to a particular concept and may or may not lexicalize (or include) properties of that concept. At the same time, many lexical items do not point to any concept and simply lexicalize properties of another concept (i.e., there is not a one-to-one mapping between concepts and lexical items in either direction). To look at a simple example, (26a) makes grammatically overt the fact that the instrument for the motion is a bicycle, while (26b) lexicalizes both the motion-event itself and the instrument involved in a single verb. Both have the same utterance meaning. ${ }^{11}$ I want to illustrate the relationship between concepts and lexical items by looking at two different semantic phenomena: parametric verbs and displaced modifiers (Nirenburg and Raskin 2004).

\section{(26a) I rode to the store on a bicycle.}

(26b) I bicycled to the store.

Parametric verbs (such as expect, try, fail, hope, and fear) are syntactic verbs which do not represent events. Thus, these lexical items do not point to a particular concept but rather modify an already instantiated concept elsewhere. Parametric verbs often add a modality or aspect to a different verb in the sentence. The point here is that these syntactic main verbs do not contain or even modify the event itself, but rather express the speaker's perception of the event. Let's look at some examples. In (27a) the main syntactic verb is expect, but the event is contained in the noun gains. This can be paraphrased as (27b). The example is especially interesting because of the disconnect between syntactic structure and utterance meaning.

10 This is similar to the position in LCCM theory (Evans 2010) which divides its representation of conceptual structure (in OnSe, the ontology) and semantic structure (in OnSe, the lexicon), with the result that many open-class lexical items have access to multiple areas of the conceptual system. Research in the OnSe paradigm has pointed out the practical difficulty of separating which of these two resources should store or describe a particular piece of content (is it linguistic or conceptual; Nirenburg and Raskin 2001)? Their answer is practical: it is a matter of engineering standards. This is required, of course, because OnSe has been implemented with full coverage, rather than with toy models and example content. 11 They do, however, provide a different prominence to the elements (e.g., [9a] focuses on the bicycle) which in many discourse contexts may have further implications. 
(27a) You should expect some gains from bonds moving forward.

(27b) Bonds may increase moving forward. You should think this is likely.

In (28a) the status of try is again not an event in itself but rather a modification of the event pointed to by continue. In (28b) the utterance meaning changes so that the action is carried out rather than simply attempted: they do continue their tactics. Thus, try represents a modality expressing someone's perceptions of the event's success or failure.

(28a) That could embolden politicians to try to continue their delaying tactics.

(28b) That could embolden politicians to continue their delaying tactics.

Displaced modifiers are adjectives that do not modify the phrase they are syntactically apart of. These are like parametric verbs in the sense that they modify another element in the utterance rather than their syntactic heads in the surface structure. In (29a) occasional again modifies the utterance meaning in terms of its aspect: the whole utterance meaning occurs repeatedly but infrequently. In (29b), though, the adjective modifies only the concept LIZARD itself.

(29a) The occasional lizard would rustle in the bushes.

(29b) The skink lizard would rustle in the bushes.

The same generalizations hold true for former in (30a): this does not change anything about the vice-chairman, rather saying that he is no longer in that position. By contrast, in (30b) the modifier most corrupt does modify the attributes of the chairman.

(30a) Miller is the former Chase vice-chairman.

(30b) Miller is the most corrupt Chase vice-chairman.

One piece of evidence for this brief analysis is that we can find minimal pairs for lexical items that point to the same concept while lexicalizing different properties of that concept. Examples (31a) and (31b) show a minimal pair for distance: (a) is longer in distance than (b) but all other specifications are the same. In (32a) and (32b) the motion is the same but the modality applied to that motion changes: one is allowed and the other is not. In (33a) and (33b) the motion differs only in velocity: one is slow and the other is fast. In (34a) and (34b) the motion differs only in the direction taken, one upward and the other downward. In (35a) 
and (35b) the motion differs only in the level of secrecy: the first is fully visible but the second is done covertly.

(31a) The girl leapt across the hole.

(31b) The girl hopped across the hole.

(32a) The prisoner exited from her cell.

(32b) The prisoner escaped from her cell.

(33a) The children walked home.

(33b) The children ran home.

(34a) The expedition ascended the mountain.

(34b) The expedition descended the mountain.

(35a) The neighbor walked through the backyard.

(35b) The neighbor stole through the backyard.

To conclude, I want to list the ways in which two lexical items can differ. Each of these different relationships between a lexical item and a concept can play a role in forced metaphorization, as we will see shortly. ${ }^{12}$

- They can point to different concepts.

- They can point to different instances of the same concept.

- One can point to a concept and the other can point to no concept. ${ }^{13}$

- They can point to the same concept but lexicalize different properties.

- They can modify entirely different parts of the utterance meaning

(e.g., occasional vs. skink in (29) above).

- $\quad$ They can point to overlapping but not identical association areas. ${ }^{14}$

12 In OnSe, different senses of a lexical item often point to different concepts; the list here assumes that the sense of the lexical item has been disambiguated so that only one concept is pointed to. In LCCM, a lexical concept is connected to or facilitates access to the conceptual structure. Although the lexical concept can facilitate access to a many different association areas, during interpretation that semantic potential is not fully activated. In other words, the list here can be formulated as the relationship between a lexical concept (after lexical concept selection) and the conceptual content to which it provides access or activates in a particular utterance (i.e., under a particular interpretation), even though the lexical concept would have different relationships with the conceptual content in other uses (i.e., under another interpretation).

13 Lexical items which point to no concept or point to a concept with certain properties show that there is a continuum between lexical and grammatical words. I am being explicit here because many lexical words exhibit properties of grammatical words, and this terminology is necessary before the predictions can be formulated.

14 This last point is true in LLCM but not in OnSe. In descriptive terms (i.e., how to represent a particular semantic phenomenon) these cases are represented using lexicalized properties and attributes in OnSe (a less cognitively real but more implementable approach). 


\subsection{Observing changes in utterance meaning}

When we try to force metaphorization by substituting a lexical item into a nonmetaphoric utterance three distinct outcomes occur:

i. the utterances have equivalent essential or non-essential meanings

ii. the utterances have different essential or non-essential meanings

iii. no interpretation is possible

When no interpretation is possible there are two reasons: (a) metaphorization has been blocked by external factors (for example, one of the utterances is conventional or idiomatic, or the substituted word does not fit into the original utterance's construction frame); (b) no metaphoric interpretation is possible. For (b) it is interesting to ask what constraints exist on possible metaphors and what the nature of those constraints are. Approaches to metaphor within cognitive linguistics predict that such constraints will exist; the existence of these constraints is a further argument against Davidson's position, to the extent that they are operative. At the same time, the constraints are somewhat problematic. For example, Barnden (2010) questions the domain approach to metaphors because it seems that metaphoric connections can be made between concepts within a single domain. In other words, it is not clear how similar two concepts can be and still be able to be metaphorically connected: perhaps metaphor does not require any domain separation. This is especially problematic when trying to separate metaphoric and metonymic utterances. In some cases, forced metaphorization results in the creation of a metonymic utterance rather than a metaphoric utterance. We can avoid this complication by choosing to substitute lexical items that create metaphoric expressions.

When forced metaphorization is possible there are two levels of meaning change: on the one hand, some express the same essential meaning (but with a difference in meaning somewhere else), and on the other hand some express different essential meanings. We can test for equivalence of this sort of meaning by finding a strong implication that differs from one version to another. A strong implication is not strictly speaking an entailment; rather, it is an implication that is not easily canceled (non-defeasible). In other words, if substitution changes one of these strong implications then we know that the essential meaning has also changed. Example (36a) has the strong implication given in (36b). Examples (36c) and (36d) have the same strong implication and thus express the same essential meaning.

(36a) Stocks tumbled on the final day of the week.

(36b) Strong Implication: Stocks are lower now than they were before. 
(36c) Stocks dived on the final day of the week.

(36d) Stocks plummeted on the final day of the week.

Let's look at some substitutions which do not have the same essential meaning. Example (37a) has the strong implication given in (37b). This example does not indicate substantial change upward or downward, but rather strongly implies that, despite minor changes, stocks have remained at the same general level. The substitution in (37c) shares this strong implication, but the substitution in (37d) does not and thus has a different essential meaning.

(37a) Stocks ambled on the final day of the week.

(37b) Strong Implication: Stocks may or may not be lower now than they were before.

(37c) Stocks drifted on the final day of the week.

(37d) Stocks sky-rocketed on the final day of the week.

Example (38a) has the strong implication in (38b.a) and the weak implication in (38b.b). I am distinguishing strong implications and weak implications here by the use of the negation test. In other words, (38b.a') is unacceptable, but (38b.b') is possible. Thus, the use of dive in requires that congress debated and was involved in the issue, but the choice could simply be that congress can no longer avoid the issue and so wishes to get through it as quickly as possible. The substitutions in (38c) through (38e) carry with them the same strong implications and the same weak implications. The conclusion, then, is that they express both the same essential meaning and non-essential meaning. Interestingly, (38c) and (38e) add more weak implications without removing the previous weak implication.

(38a) Congress dived into a controversial topic last tonight.

(38b.a) Strong Implication: Congress debated and tried to resolve a controversial topic.

(38b.a') ${ }^{*}$ Congress did not debate or try to resolve the topic, however.

(38b.b) Weak Implication: Congress is active or purposeful in doing this.

(38b.b') Congress is not being courageous: they can't avoid it any longer.

(38c) Congress galloped into a controversial topic last tonight.

(38d) Congress jumped into a controversial topic last tonight.

(38e) Congress strutted into a controversial topic last tonight.

Let's look at a series of examples which changes the strong implications. In (39a) the use of amble reverses the strong implication to that in (39b.a), as shown 
by the fact that the negation of the strong implication in (39b.a') is unacceptable. While (39a) weakly implies (39b.b), the negation of the weak implication does not produce an unacceptable statement, showing that this is not a strong implication as I am using the term. Examples (39c) through (39e) do not change either the strong implication or the weak implication of the original metaphor.

(39a) Congress wandered into a controversial topic last tonight.

(39b.a) Strong Implication: Congress had not planned this topic in this way.

(39b.a') *Congressmen had been preparing their positions for months in advance.

(39b.b) Weak Implication: Congress is not doing this purposefully, rather accidentally.

(39b.b') Congress wants to test the issue before the midterm elections.

(39c) Congress skidded into a controversial topic last tonight.

(39d) Congress slipped into a controversial topic last tonight.

(39e) Congress strayed into a controversial topic last tonight.

It is important to note here that strong and weak implications of this sort are not restricted to metaphors and in fact are derived in much the same way in nonmetaphoric utterances. This is important because we will be using strong and weak implications to show that the output of forced metaphorization is predictable. These implications apply to metaphoric and non-metaphoric utterances in the same way because both have a direct meaning.

\subsection{Predicting meaning change during forced metaphorization}

My claim is that we can make predictions about what meaning change will occur during forced metaphorization and that these predictions are evidence for the stable interpretation of metaphoric utterances (which are themselves evidence for the existence of metaphoric meanings). We can use multiple lexical items as substitutes during forced metaphorization and then compare the essential meaning and non-essential meaning of their respective metaphoric utterances to see if they are the same or different.

Lexical items often point to concepts. (1) The first prediction is that substituting lexical items which point to the same concept will cause a similar change in essential meaning during forced metaphorization. (2) The second prediction is that substituting lexical items which point to the same concept but have different lexicalized properties will have similar changes in essential meaning during forced metaphorization but different changes in non-essential meaning. (3) The third prediction is that substituting lexical items which point to 
different concepts will cause different changes in essential meaning during forced metaphorization.

These are not complicated predictions. They do not need to be. They only need to be valid to show that metaphors (by which I mean low metaphoricity and unsaturated metaphoric utterances) have a stable interpretation. As discussed above, we can use strong implications and weak implications to decide whether the output of forced metaphorization with two different lexical items have the same essential meaning or the same non-essential meaning. Thus, we do not need to worry about how to represent the two sorts of meaning for these metaphoric utterances. We only need to find out if meaning of two different utterances created through forced metaphorization are or are not equivalent. ${ }^{15}$

\subsection{What happens during forced metaphorization}

The output of forced metaphorization is a metaphoric interpretation of the utterance meaning as a whole, not necessarily the meaning of the individual lexical item that was substituted into the utterance. At the same time, we know that the only difference in the utterances are their respective lexical items (the original and its substitute). The substituted lexical item itself does not contain a metaphoric meaning, as if placing a metaphoric word into an utterance makes the whole utterance instantly metaphoric by its mere presence. Rather, the relation between the substituted lexical item and the rest of the utterance forces or prompts a metaphoric reading. This happens because the constructional context (surrounding arguments) "provide the direct link between surface form and general aspects of the interpretation” (Goldberg 2009: 95). In other words, the main verb (which is what we are substituting here) underspecifies the structure of the clause, the construction in which the utterance is presented. Because of this, the other arguments have an important role in shaping the construction, not simply serving as slots for the substituted verb. It is this interaction between the substituted verb and the rest of the construction that coerces or shapes the nonmetaphoric meaning of the utterance and gives rise to a direct metaphoric interpretation. In short, although prompted by the substitution of a lexical item, forced metaphorization affects the utterance as a whole.

15 Non-metaphoric utterances, of course, can have similarly stable meanings and implications after substitution in this way. The point here is not that metaphoric utterances differ from non-metaphoric utterances in this respect, but rather that they are the same. The fact that they have similar properties is because these sorts of metaphors have a direct meaning that is stable and consistently interpreted. 
We can describe the process in more detail by adopting the framework of LCCM theory (Evans 2010). The conception (i.e., utterance-meaning) of the original sentence undergoes lexical content selection which disambiguates which lexical concepts are involved. These lexical concepts are fused into the final meaning by first integrating the lexical contents into a construction and then activating the parts of the conceptual system which they point to or access. The activation of the conceptual system is constrained by the relations between the lexical concepts, including the matching of the lexical concepts to one another based on certain properties (not unlike a nuanced implementation of selectional restrictions: Katz and Fodor 1963). In the original non-metaphoric utterance, the match will occur within the primary cognitive models and the interpretation will be complete. In the metaphoric utterances, the match will not occur here and the search will extend to the secondary cognitive models until a match occurs and interpretation is possible. Thus, the divergence between the original utterance and the metaphorized utterances is at the point of matching the primary cognitive models, and the divergence between different metaphorized utterances is at the point of searching for a match beyond the primary models. The prediction is that lexical content which accesses the same areas of the conceptual structure will find a match in the same secondary cognitive model and thus will produce an interpretation with the same essential meaning.

\subsection{Forced metaphorization with lexical items that point to the same concept}

The prediction is that if we take a non-metaphoric utterance and force it to become metaphoric by substituting a particular lexical item into the utterance, then lexical items which point to the same concept and have similar lexicalized meanings will share the same essential meanings. Let's look at some examples. The original non-metaphoric utterance in (40a) has the verb ignore which points to a concept which we can call communicATION. The substituted verbs in (40b-d) are flee, evade, and abandon, all of which point to the concept which we can call MOTION. Further, each of these substituted verbs lexicalizes similar meaning: the motion is away from a particular source with an intention to leave the source behind. Thus, these lexical items point to the same concept with roughly similar lexicalized meaning. We can make a strong implication that all three of the metaphorized utterances share in (40e), so that they all share the same essential meaning. This also aligns with our intuitions. Thus, the same concept is lexicalized in each substituted verb and each metaphoric utterance has a similar essential meaning. 
(40a) Non-metaphoric: Irrational people ignore scientific reasoning.

(40b) Metaphorized 1: Irrational people flee scientific reasoning.

(40c) Metaphorized 2: Irrational people evade scientific reasoning.

(40d) Metaphorized 3: Irrational people abandon scientific reasoning.

(40e) Strong Implication: Irrational people do not make conclusions based on scientific reasoning.

The non-metaphoric utterance (41a) has the verb made which points to a concept which we can call CREATE. The substituted verbs chased, entered, and reached in (41b-d) all share the same concept which we can again call MOTION, and each lexicalizes similar properties of this concept: this movement is towards a particular goal and the intention is to reach that goal. We can make a strong implication that each of these metaphorized utterances shares in (41e) which reinforces our intuitions that each of the metaphorized utterances has the same essential meaning. This, again, matches our predictions.

(41a) Non-metaphoric: China has made a trade agreement with the European Union.

(41b) Metaphorized 1: China has chased a trade agreement with the European Union.

(41c) Metaphorized 2: China has entered a trade agreement with the European Union.

(41d) Metaphorized 3: China has reached a trade agreement with the European Union.

(41e) Strong Implication: China and the European Union now have a trade agreement with one another.

\subsection{Forced metaphorization with lexical items that point to the same concept with different lexicalized meanings}

The prediction is that if we take a non-metaphoric utterance and force it to become metaphoric by substituting a particular lexical item into the utterance, then lexical items which point to the same concept but have different lexicalized meanings will share the same essential meanings but differ in their non-essential meanings. Let's look at some examples. In (42b-d) the substituted lexical items meandered, sauntered, and struggled all point to the same concept of MотіоN, but each lexicalizes a different meaning. Meandered lexicalizes the lack of a specific destination and the slow speed of the movement. Sauntered lexicalizes the manner of the movement, a slow and assured manner, and does not specify the desti- 
nation or lack of destination. Struggled lexicalizes the difficult manner of the motion, that it is somehow impeded. Each of these shares the strong implication in (42e), which corresponds with our intuitions that they have the same essential meaning. However, they do not share the same weak implications which means that they do not share the same non-essential meaning. The use of meandered in (42b) has the weak implication that Jason does not have a fixed point, that he is wandering in his speech. The use of sauntered in (42c) has the weak implication that Jason is speaking slowly but with a certain amount of self-assuredness. The use of struggled in (42d) has the weak implication that Jason's voice or his speech is having trouble being understood. The fact that each of these metaphorized utterances has a different implication is evidence that they do not share the same non-essential meaning. This matches our prediction because we expect that different lexicalized meanings pointing to the same concept will differ in this way.

(42a) Non-metaphoric: Jason's voice sounded over the telephone line.

(42b) Metaphorized 1: Jason's voice meandered over the telephone line.

(42c) Metaphorized 2: Jason's voice sauntered over the telephone line.

(42d) Metaphorized 3: Jason's voice struggled over the telephone line.

(42e) Strong Implication: Jason's voice could be heard through the telephone.

The three substituted verbs in (43b-d) are stampeded, wandered, and crawled which again all share the same concept (MOTION) but differ in the lexicalized meaning which they add to that concept. All three metaphorized utterances share the strong implication in (43e), which corresponds with our intuitions that they share the same essential meaning. Each, however, has a different weak implication. Stampeded lexicalizes the manner of the motion, that it occurs forcefully, as a mass. The weak implication in this utterance is that these investors were not necessarily welcome but beat back opposition. Wandered lexicalizes that there is no specific destination for this motion. The weak implication in this utterance is that these investors do not have a clear plan for their involvement and did not all invest at the same time or at the same pace. Crawled lexicalizes the manner of motion and, as a result, its speed: slow. The weak implication in this utterance is that the investors moved slowly and carefully as they entered these markets. Thus, again, these lexical items point to the same concept but have different lexicalized meanings and, during forced metaphorization, they produce a similar change in essential meaning but have different changes in non-essential meaning. This matches our earlier predictions.

(43a) Non-metaphoric: They bought into emerging markets when interest rates were low. 
(43b) Metaphorized 1: They stampeded into emerging markets when interest rates were low.

(43c) Metaphorized 2: They wandered into emerging markets when interest rates were low.

(43d) Metaphorized 3: They crawled into emerging markets when interest rates were low.

(43e) Strong Implication: They are now invested in these emerging markets.

\subsection{Forced metaphorization with lexical items that point to different concepts}

The prediction is that if we take a non-metaphoric utterance and force it to become metaphoric by substituting a particular lexical item into the utterance, then lexical items which point to the different concepts will have different essential and non-essential meanings. Let's look at some examples. The three substituted verbs in $(44 \mathrm{~b}-\mathrm{d})$ point to different concepts. Invade points to the concept MотіоN and lexicalizes that this motion is a sort of entering with a hostile intent and for the purpose of conquering. This utterance strongly implies that members of congress have entered Mexico and weakly implies that they are not welcome or that their intent is malicious. Brandished points to the concept HOLD and lexicalizes that what is held is possibly a weapon that is being waved about in a threatening manner. This utterance strongly implies that members of congress are using Mexico for some sort of purpose during a fact-finding mission. It weakly implies that their use of Mexico is threatening in some way, or for dramatic display. Lampooned points to the concept CommunicATION and lexicalizes that the communication is intended to criticize or satirize a particular target. This utterance strongly implies that members of congress criticized Mexico in some way on this mission. It weakly implies that they did so in an unbecoming manner. Thus, we see that using lexical items that point to different concepts causes differences in essential meaning and non-essential meaning. This matches our predictions.

(44a) Non-metaphoric: Members of congress entered Mexico while on a factfinding mission.

(44b) Metaphorized 1: Members of congress invaded Mexico while on a factfinding mission.

(44c) Metaphorized 2: Members of congress brandished Mexico while on a factfinding mission.

(44d) Metaphorized 3: Members of congress lampooned Mexico while on a factfinding mission. 
The three substituted lexical items in $(45 b-d)$ again point to different concepts. Hijacked points, perhaps, to the concept GAIN CONTROL and lexicalizes that such control is opposed and that it is gained by using illegitimate means. This utterance strongly implies that the secret papers have gained control over the internet in some way. The weak implication is that this control is not beneficial, that the papers are diverting attention from other matters. Ignited points to the concept BURN and lexicalizes that the agent is causing something to burn. The strong implication in this utterance is that the secret papers have become spread all over the internet. The weak implication is that the reaction of those who use the internet to these papers is furious opposition or disgust. Resurrected points to the concept LIVE and lexicalizes that the agent is causing the theme to come to life again. The strong implication here is that the internet was reenergized by the release of the secret papers. The weak implication is that the internet was in some way diminishing before this happened. So, again, these lexical items point to different concepts and thus the metaphoric utterances they create have different essential meanings.

(45a) Non-metaphoric: The secret papers slowly entered the internet.

(45b) Metaphorized 1: The secret papers slowly hijacked the internet.

(45c) Metaphorized 2: The secret papers slowly ignited the internet.

(45d) Metaphorized 3: The secret papers slowly resurrected the internet.

\subsection{Conclusions}

The testable predictions made about the interpretations of metaphoric utterances, which adhere to the highest standard of falsifiability, were not falsified. The predictions matched the actual interpretations of metaphoric utterances. This fact results in a plausible inference that these metaphoric utterances have a stable interpretation. I have argued in Section 2 that some, but not all, metaphoric utterances have a direct meaning. The stable interpretations shown in Section 3 are evidence for this direct meaning. This is significant for three reasons: First, it provides a new source of evidence that largely converges with prior evidence from within the cognitive linguistics paradigm. In this sense it strengthens the cognitive linguistic position. Second, it adds nuances to that position: the linguistic structure of the metaphoric utterance influences the interpretability of that utterance. In this sense it expands the cognitive linguistic position from metaphor-in-thought to metaphor-in-language. Third, it opens the possibility of reconciling approaches to metaphor within cognitive linguistics 
with those in formal semantics and, possibly, relevance theory. If linguistic (and other) properties can be used to divide metaphors into a number of sub-groups, then the scope of different approaches to metaphor can be tied directly to a particular sub-group. Davidson is right about some metaphors; cognitive linguistics is right about some metaphors. This enhances the descriptive coverage of metaphor as a whole while also working toward the larger reconciliation of different approaches to meaning in language (a goal whose benefits I take as self-evident).

\section{References}

Bakhtin, Michail. 1994 [1930s]. The dialogic imagination: Four essays. Holquist, Michael (ed.), Emerson, Caryl \& Holquist, Michael (translators). Austin: University of Texas Press.

Barnden, John. 2001. Application of the ATT-Meta metaphor-understanding approach to various examples in the ATT-Meta project databank. Technical report CSRP-01-02. Birmingham, UK: School of Computer Science, University of Birmingham.

Barnden, John. 2010. Metaphor and metonymy: Making their connections more slippery. Cognitive Linguistics, 21(1), 1-34.

Bezuidenhout, Anne. 2001. Metaphor and what is said: A defense of a direct expression view of metaphor. Midwest studies in philosophy, 25(1), 156-186.

Black, Max. 1962. Models and metaphors: Studies in language and philosophy. Ithaca: Cornell University.

Black, Max. 1977. More about metaphor. Dialectica, 31(3/4), 431-457.

Black, Max. 1979. How metaphors work: A reply to Donald Davidson. Critical Inquiry, 6(1), 131-143.

Coulson, Seana \& Matlock, Teenie. 2001. Metaphor and the Space Structuring Model. Metaphor \& Symbol, 16(3/4), 295-316.

Davidson, Donald. 1978. What metaphors mean. Critical Inquiry, 5(1), 31-47.

Davies, Mark. 2008. The Corpus of Contemporary American English: 450 million words, 1990-present. http://corpus.byu.edu/coca/ [accessed August 2012].

Dirven, René. 1993. Metonymy and metaphor: Different mental strategies of conceptualization. Leuvense Bijdragen, 82, 1-28.

Dunn, Jonathan. 2011. Gradient semantic intuitions of metaphoric utterances. Metaphor \& Symbol, 26(1), 53-67.

Evans, Vyvyan. 2010. Figurative language understanding in LCCM Theory. Cognitive Linguistics, 21(4), 601-662.

Falck, Marlene Johansson \& Gibbs, Raymond Jr. 2012. Embodied motivations for metaphorical meanings. Cognitive Linguistics, 23(2), 251-272.

Fillmore, Charles. 1967. The case for case. In Bach, Emmon \& Harms, Robert (eds.), Universals in linguistic theory. New York: Holt, Rinehart, and Winston, 1-88.

Goldberg, Adele. 2009. The nature of generalization in language. Cognitive Linguistics, 20(1), 93-127.

Grady, Joseph. 2007. Metaphor. In Geeraerts, Dirk \& Cuyckens, Hubert (eds.), The Oxford handbook of cognitive linguistics. Oxford, UK: Oxford University Press, 188-213. 
Katz, Jerrold \& Fodor, Jerry. 1963. The structure of a semantic theory. Language 39(2), $170-210$.

Kertész, András \& Rákosi, Csilla. 2009. Cyclic vs. circular argumentation in the Conceptual Metaphor Theory. Cognitive Linguistics, 20(4), 703-732.

Kövecses, Zoltán. 2010. A new look at metaphorical creativity in cognitive linguistics. Cognitive Linguistics, 21(4), 663-697.

Lakoff, George. 1987. Women, fire, and dangerous things: What categories reveal about the mind. Chicago: University of Chicago Press.

Lakoff, George \& Johnson, Mark. 1980. Metaphors we live by. Chicago: University of Chicago Press.

Lakoff, George \& Johnson, Mark. 1999. Philosophy in the flesh: The embodied mind and its challenge to western thought. New York: Basic Books.

Lee, Mark \& Barnden, John. 2001. Reasoning about mixed metaphors with an implemented AI system. Metaphor \& Symbol, 16(1/2), 29-42.

Martinich, Aloysius. 1984. A theory for metaphor. Journal of Literary Semantics, 13(1), 35-56.

Narayanan, Srinivas. 1999. Moving right along: A computational model of metaphoric reasoning about events. Proceedings of the National Conference on Artificial Intelligence (AAAl-99), 121-127. Orlando, Florida, July 18-22, 1999.

Nirenburg, Sergei \& Raskin, Victor. 2001. Choices for lexical semantics. Computational Intelligence, 17(1), 157-177.

Nirenburg, Sergei \& Raskin, Victor. 2004. Ontological semantics. Cambridge: M. I. T. Press.

Panther, Klaus-Uwe \& Thornburg, Linda. 1998. A cognitive approach to inferencing in conversation. Journal of Pragmatics 30(6), 755-769.

Popper, Karl. 1959 [1934]. The logic of scientific discovery. New York: Basic Books.

Reimer, Marga. 2001. Davidson on metaphor. Midwest Studies in Philosophy, 25(1), 142-155.

Ruiz de Mendoza, Francisco \& Perez Hernandez, Lorena. 2011. The contemporary theory of metaphor: Myths, developments and challenges. Metaphor \& Symbol, 26(3), 161-185.

Searle, John. 1979. Metaphor. In Ortony, Andrew (ed.), Metaphor and Thought, 92-123. Cambridge, UK: Cambridge University Press.

Wearing, Catherine. 2006. Metaphor and what is said. Mind \& Language, 21(3), 310-332. 


\section{Appendix 1: Verb List}

PHYSICAL

butcher

detach

jump

migrate

throw

MENTAL

convince

enjoy

imagine

offend

see

SOCIAL

buy

marry

neglect

obey

punish

ABSTRACT

cipher/decipher

compute

invest

program

subtract

\section{Acknowledgements}

I am grateful for insightful discussions on this research with Victor Raskin, Julia Taylor, and Michael Maune and for the very helpful comments on earlier versions of this paper by three anonymous referees and the editor. 